\title{
Increased inflammation, endoplasmic reticulum stress and oxidative stress in endothelial and macrophage cells exacerbate atherosclerosis in ApoCIII transgenic mice
}

\author{
Han Yingchun ${ }^{1}$, Ma Yahong ${ }^{2}$, Wen Jiangping ${ }^{3}$, He Xiaokui ${ }^{3}$ and Zhang Xiaohong ${ }^{3^{*}}$
}

\begin{abstract}
Background: Overexpression of apolipoprotein CIII (ApoCIII) leads to hypertriglyceridemia (HTG) which promotes atherosclerosis development. However, it remains unclear whether ApoCIII affects the atherosclerosis alone by promoting the inflammation and endoplasmic reticulum (ER) stress, or in combination with HTG.

Methods: Transgenic (ApoCIIltg) mouse models were used to investigate the atherogenic role of ApoCIII. Since endothelial cells and macrophages play crucial roles in atherosclerosis, we examined whether triglyceride-rich lipoproteins (TRLs), the major lipoproteins, in plasma of ApoCIlltg mice affect inflammation and ER stress levels in these cells. To further investigate the role of ApoCIII and triglyceride, we incubated HUVECs cells and peritoneal macrophages with TRLs with or without ApoCIII.

Results: Increased inflammation and ER stress were found in the aorta of ApoCllltg mice. TRLs increased ER stress and oxidative stress in HUVECs and macrophages in a dose dependent. Moreover, TRLs together with ApoCIII could induce a higher inflammation level than TRLs alone in these cells.
\end{abstract}

Conclusions: Both TRLs and ApoCIII contribute to the progression of atherosclerosis, and the modulation of TRLS and ApoCIII may represent a novel therapeutic approach against HTG induced atherosclerosis.

Keywords: Apolipoprotein CIII, Triglyceride-rich lipoprotein, Endothelial cells, Macrophages, Inflammation, Endoplasmic reticulum stress, Oxidative stress

\section{Background}

Cardiovascular disease is a major cause of death globally. Atherosclerosis begins with vascular endothelial dysfunction, activation and recruitment of monocytes to the vascular wall, differentiation into macrophages, uptake of cholesterol and other lipoproteins, and formation of foam cells [1]. It is widely known that LDL, especially oxidative LDL, plays a major role in the initiation and development of atherosclerosis, and the molecular mechanism has also been studied extensively [2]. However, in

\footnotetext{
*Correspondence: zhxhzhxh2002@163.com

${ }^{3}$ Department of Laboratory Medicine, Beijing Tongren Hospital, Capital Medical University, Beijing 100730, China

Full list of author information is available at the end of the article
}

recent years, it has become more evident that postprandial hyperlipidemia, large numbers of triglyceride-rich lipoproteins (TRLs), is also a significant risk factor for atherosclerotic cardiovascular disease [3-5]. Emerging evidence has shown that postprandial hyperlipidemia is a pro-inflammatory factor and TRLs participate in a large number of inflammation related processes, including excessive free radical production, leukocyte activation, endothelial dysfunction and the formation of foam cells $[6,7]$.

Regulation of plasma triglyceride homeostasis relies on a variety of enzymes and proteins including lipoprotein lipase (LPL), hepatic lipase, ApoCIII, ApoCII, and ApoAV [8]. An important regulator of triglyceride metabolism, the glycoprotein ApoCIII contains 79 amino acids,

(c) The Author(s). 2018 Open Access This article is distributed under the terms of the Creative Commons Attribution 4.0 International License (http://creativecommons.org/licenses/by/4.0/), which permits unrestricted use, distribution, and reproduction in any medium, provided you give appropriate credit to the original author(s) and the source, provide a link to the Creative Commons license, and indicate if changes were made. The Creative Commons Public Domain Dedication waiver (http://creativecommons.org/publicdomain/zero/1.0/) applies to the data made available in this article, unless otherwise stated. 
and is mainly synthesized by the liver and small intestine [9]. Plasma ApoCIII could inhibit LPL activity and interfere the liver uptake of TRLs by receptor dependent and independent pathways [10]. In addition, ApoCIII promotes the assembly and secretion of VLDL in the liver [11]. ApoCIII mainly distributes in TRLs at hypertriglyceridemia condition.

Surprisingly, in vitro experiments from our group [12] and others show that ApoCIII directly promotes the expression and activation of VCAM-1 in HUVECs and induces monocyte adhesion, thereby increasing the inflammatory response [10-12], indicating the significant effect of ApoCIII protein. Furthermore, two large genetic studies, one conducted in Denmark on people of mainly European origin [13] and the other in the United States on people of European and African origin [14], showed that people with loss-of-function mutations in the ApoCIII gene have a significantly reduced risk of having atherosclerotic cardiovascular disease.

Recently, we showed that ApoCIII induces hypertriglyceridemia which leads to aggravation of aortic atherosclerosis. Increased inflammation and oxidation level in vascular smooth muscle cells (VSMCs) play an important role in atherosclerosis progression [12]. Since both endothelial cells and macrophages are also involved in the initiation and development of atherosclerosis [15], we investigated the effect of TRLs to endothelial cells and macrophages and demonstrated the effect of triglycerides and ApoCIII in this process.

\section{Materials and methods}

\section{Animals}

The human ApoCIIItg, ApoCIII-/-, LDLR-/- and GPIHBP1-/- mouse models were purchased from the Jackson Laboratory (Bar Harbor, ME, USA). The study was approved by the Animal Care Committee of Peking University Health Science Center and in agreement with the Guide for the Care and Use of Laboratory Animals published by the US National Institutes of Health (NIH Publication, 8th Edition, 2011).

The ApoCIIItg/LDLR-/- and ApoCIIItg/GPIHBP1-/mice were generated by crossing ApoCIIItg mice with LDLR-/- and GPIHBP1-/- Mice, respectively. The ApoCIII/GPIHBP1 DKO mice were generated by crossing ApoCIII-/- with GPIHBP1-/- mice.

Eight-week old male LDLR-/- (controls) and ApoCIIItg/LDLR-/- mice were fed with a high-fat, cholesterol-rich diet (20\% lard and $0.5 \%$ cholesterol) for 3 months to develop atherosclerosis.

\section{Tissue harvesting and processing}

After 3 months of pro-atherogenic diet, LDLR-/(controls) and ApoCIIItgLDLR-/- mice were sacrificed. After the left ventricle after a right atrium cut was rinsed slowly with $1 \times$ phosphate-buffered saline (PBS), the aorta was harvested for Western blotting. The heart was post-fixed with $4 \%$ paraformaldehyde for $2 \mathrm{~h}$ and immersed in $30 \%$ sucrose overnight. The top part of the heart was embedded in OCT, snap-frozen in liquid nitrogen, and stored at $-80{ }^{\circ} \mathrm{C}$ prior to sectioning. $10 \mu \mathrm{m}$ cryosections of aortic sinus sections were then subjected to immunohistochemistry and ORO staining.

\section{Isolation of TRLs}

Plasma of heparin-treated whole blood from ApoCIIItg/ GPIHBP1-/- and ApoCIII/GPIHBP1 DKO mice was separated by centrifugation $\left(4000 \mathrm{rpm}, 4{ }^{\circ} \mathrm{C}, 10 \mathrm{~min}\right)$. TRLs, consisting of CMs and VLDLs, were isolated from plasma by ultracentrifugal spin $\left(42,000 \mathrm{rpm}, 10{ }^{\circ} \mathrm{C}, 3 \mathrm{~h}\right)$ at density $1.006 \mathrm{~g} / \mathrm{ml}$ in a Hitachi P42AT rotor. Triglyceride contents in TRLs were measured by a kit from Sigma (TR0100).

\section{Cell culture}

HUVECs cells were isolated from umbilical cords by collagenase digestion and cultured on plates coated with $50 \mathrm{mg} / \mathrm{ml}$ collagen as described [12]. Cells were maintained in M-199 medium supplemented with $20 \mathrm{mmol} / \mathrm{L}$ HEPES, pH 7.4, 20\% fetal bovine serum (FBS), $5 \mathrm{ng} / \mathrm{ml}$ recombinant human fibroblast growth factor, antibiotics/ antimycotics, and $90 \mathrm{mg} / \mathrm{ml}$ heparin (EC medium). Passages 3 to 5 were used and cell were treated when HUVECs were grown to $70-80 \%$ confluency. HUVECs were starved for $4 \mathrm{~h}$ before adding TRLs into the medium. For inflammation, ER stress or oxidative stress, HUVECs were treated with TRLs $(100 \mu \mathrm{g} / \mathrm{ml}$ TG concentration or indicated concentration) for 24 or $48 \mathrm{~h}$, and the cells were harvested. For inflammation, $1 \mu \mathrm{g} / \mathrm{ml}$ lipopolysaccharide (LPS) was added $6 \mathrm{~h}$ before RNA or protein extraction.

Female BALB/c mice (8-14 weeks of age) were intraperitoneally administered $2 \mathrm{ml}$ of a 4.05\% solution of Thioglycolate (Sigma, USA). Four days later, the mice were euthanized, and the peritoneal macrophages were collected in cold phosphate-buffered saline (PBS). The cells were cultured in Dulbecco's modified Eagle's medium (Gibco, USA) supplemented with 10\% FBS and $2 \mathrm{mM} \mathrm{l-glu-}$ tamine.Two hours later, the medium was replaced with fresh medium to remove non-adherent cells. Fresh medium was added to the wells, and the cells were cultured at $37^{\circ} \mathrm{C}$ for $24 \mathrm{~h}$. To obtain sufficient numbers of cells, macrophages were collected from 2 to 3 mice and pooled for some experiments. Macrophages were treated with TRLs $(100 \mu \mathrm{g} / \mathrm{ml}$ TG concentration) for $24 \mathrm{~h}$.

Rat VSMCs were isolated from aortas of 80-100 g male Sprague-Dawley rats anesthetized intraperitoneally with sodium pentobarbital ( $50 \mathrm{mg} / \mathrm{kg}$ body weight). Rats were humanely sacrificed by cervical dislocation in order to obtain tissues to harvest VSMCs. All VSMCs experiments were 
performed on primary culture and passages $3-5$ were used. VSMCs were treated with TRLs $(100 \mu \mathrm{g} / \mathrm{ml}$ TG concentration) for $24 \mathrm{~h}$.

\section{Immunohistochemistry (IHC)}

For IHC analysis, cryosections were fixed (10 min) in cold $4 \%$ paraformaldehyde solution and rinsed $(10 \mathrm{~min})$ with PBS (0.1 M, pH 7.4) supplemented with $3 \%$ hydrogen peroxide. After washing and incubation (30 min) in blocking solution (PBS containing 10\% goat serum), the sections were incubated overnight at $4{ }^{\circ} \mathrm{C}$ with 1:200 rabbit anti-VCAM-1 antibody (ab134047, Abcam), 1:400 rabbit anti-Mac2 antibody (sc-53,127, Santa Cruz) or 1:400 rabbit anti-4HNE antibody (ab46545, Abcam) diluted in blocking solution, washed three times with PBS, incubated with HRP-conjugated secondary antibody (for VCAM-1, Mac2, 4HNE) for $1 \mathrm{~h}$ at $37^{\circ} \mathrm{C}$, rinsed with PBS, counterstained with hematoxylin if needed and examined by light microscopy with diaminobenzidine (DAB) as chromogen.

\section{Western blot}

Cells in culture plates were washed in ice-cold PBS twice and lysed in RIPA buffer (Cell Signaling Technology, \#9806) containing complete protease inhibitor cocktail tablets (Roche). Protein lysates $(20-40 \mu \mathrm{g})$ were resolved using SDS/PAGE and transferred to nitrocellulose membranes (Millipore). Primary antibody incubations were performed at $4{ }^{\circ} \mathrm{C}$ overnight using a 1:1000 dilution for anti-VCAM-1 antibody (ab134047, Abcam), anti-ICAM-1 antibody (ab171123, Abcam), or anti-GRP78 antibody (\#3177, Cell Signaling Technology). Secondary antibody incubation was performed using a 1:5000 dilution of goat anti-rabbit HRP conjugate antibody (\#7074, Cell Signaling Technology). Protein bands were visualized by LumiGLO ${ }^{\circ}$ Reagent (\#7003, Cell Signaling Technology).

\section{RNA isolation and quantitative real-time PCR}

Total RNA from aorta and cells were extracted using Tri Reagent (Molecular Research Center), and first-strand cDNA was generated using an RT kit (Invitrogen). Quantitative real-time PCR was performed using primer sets shown in Table 1. Amplifications were performed in 35 cycles using an opticon continuous fluorescence detection system (MJ Research) with SYBR Green fluorescence (Molecular Probes, Eugene, OR). Each cycle consisted of heating denaturation for $30 \mathrm{~s}$ at $94{ }^{\circ} \mathrm{C}$, annealing for $30 \mathrm{~s}$ at $55^{\circ} \mathrm{C}$, and extension for $30 \mathrm{~s}$ at $72{ }^{\circ} \mathrm{C}$. All samples were quantified using the comparative $C_{T}$ method for relative quantitation of gene expression, normalized to $\beta$-actin.

\section{Statistical analysis}

All data are presented as mean \pm SEM. Statistical comparison between two groups was performed using Student's
Table 1 Sequences of primers used for PCR

\begin{tabular}{|c|c|}
\hline Gene & Primer sequences $\left(5^{\prime} \rightarrow 3^{\prime}\right)$ \\
\hline \multicolumn{2}{|l|}{ Human } \\
\hline MCP1 (Forward) & GCTCATAGCAGCCACCTT \\
\hline (Reverse) & GGAATCCTGAACCCACTT \\
\hline Chop (Forward) & GGAAACAGAGTGGTCATTCCC \\
\hline (Reverse) & CTGCTTGAGCCGTTCATTCTC \\
\hline HO-1 (Forward) & TTTGAGGAGTTGCAGGAGC \\
\hline (Reverse) & AGGACCCATCGGAGAAGC \\
\hline GRP78 (Forward) & TCCTATGTCGCCTTCACT \\
\hline (Reverse) & ACAGACGGGTCATTCCAC \\
\hline GRP94 (Forward) & GTTTGGTGTCGGTTCTA \\
\hline (Reverse) & GAGTGTTTCCTCTTGGGT \\
\hline$\beta$-actin (Forward) & CGTGGGCCGCCCTAGGCACCA \\
\hline (Reverse) & TTGGCCTTAGGGTTCAGGGGGG \\
\hline \multicolumn{2}{|l|}{ Mouse } \\
\hline p47 (Forward) & ACACCTTCATTCGCCATATTGC \\
\hline (Reverse) & TCGGTGAATTTTCTGTAGACCAC \\
\hline p67 (Forward) & GCTGCGTGAACACTATCCTGG \\
\hline (Reverse) & AGGTCGTACTTCTTCATTCTGTA \\
\hline NOX-2 (Forward) & CCAAGGTATCCAAGTT \\
\hline (Reverse) & TCCAGTCTCCCACAAT \\
\hline NOX4 (Forward) & TAAGCCATCACCATCAT \\
\hline (Reverse) & TGGAGGCAGTAGTAAATC \\
\hline Catalase(Forward) & AGCGACCAGATGAAGCAG \\
\hline (Reverse) & TTCCCACAAGATCCCAGT \\
\hline SOD-1 (Forward) & TCCGTCGGCTTCTCGTCT \\
\hline (Reverse) & ACCGCTTGCCTTCTGCTC \\
\hline GRP78 (Forward) & ACTTGGGGACCACCTATTCCT \\
\hline (Reverse) & GTTGCCCTGATCGTTGGCTA \\
\hline IL-6 (Forward) & TTCTTGGGACTGATGCTG \\
\hline (Reverse) & CTGGCTTTGTCTTCTTGTT \\
\hline TNF-a (Forward) & CTGTGAAGGGAATGGGTGTT \\
\hline (Reverse) & CAGGGAAGAATCTGGAAAGGTC \\
\hline IL-10 (Forward) & ACCTGGTAGAAGTGATGC \\
\hline (Reverse) & AAGGAGTTGTTTCCGTTA \\
\hline TGF $\beta-1$ (Forward) & GGCGGTGCTCGCTTTGTA \\
\hline (Reverse) & TCCCGAATGTCTGACGTATTGA \\
\hline
\end{tabular}

t-test or one-way ANOVA. A value of $P<0.05$ was considered statistically significant.

\section{Results}

Inflammation, oxidative stress and ER stress in vivo To investigate the atherogenic role of ApoCIII, ApoCIIItg/LDLR-/- and LDLR-/- littermates were fed with an atherogenic diet for 12 weeks. Atherosclerotic lesion size in the aorta root was then measured by ORO 
staining. Consistent with our previous report [12], ApoCIII increases atherosclerotic lesions. Immunohistochemistry (IHC) staining for Mac2, a macrophage marker, indicated an increased macrophage infiltration in the lesion area. Staining of $4 \mathrm{HNE}$, the product of lipid peroxidation that can be used to evaluate the oxidation level, showed that lipid peroxidation accumulated in the aorta of ApoCIIItg mice (Fig. 1a). IHC staining and Western blotting showed that VCAM-1 and ICAM-1 increased in aorta suggesting elevated inflammation (Fig. 1b). ER stress is involved in the initiation and development of atherosclerosis. We also detected that GRP78, an important chaperone molecule related to ER stress, significantly increases in the aorta (Fig. 1c).
Thus, compared to the control LDLR-/- littermates, ApoCIIItg/LDLR-/- mice showed increased inflammation, oxidative stress and ER stress in local aorta.

\section{Inflammation in HUVECs and macrophages}

Atherosclerosis is an inflammatory disease, starting from endothelial dysfunction, infiltration of macrophages in the vessel wall, and then the formation of foam cells [15]. ApoCIII transgene increased infiltration of macrophages and VCAM-1 expression, suggesting increased inflammation (Fig. 1a and b). It is reported that ApoCIII and VLDL containing ApoCIII could promote the expression of VCAM-1 and increase the adhesion of monocytes via a PKCbeta and NF-kB dependent pathway

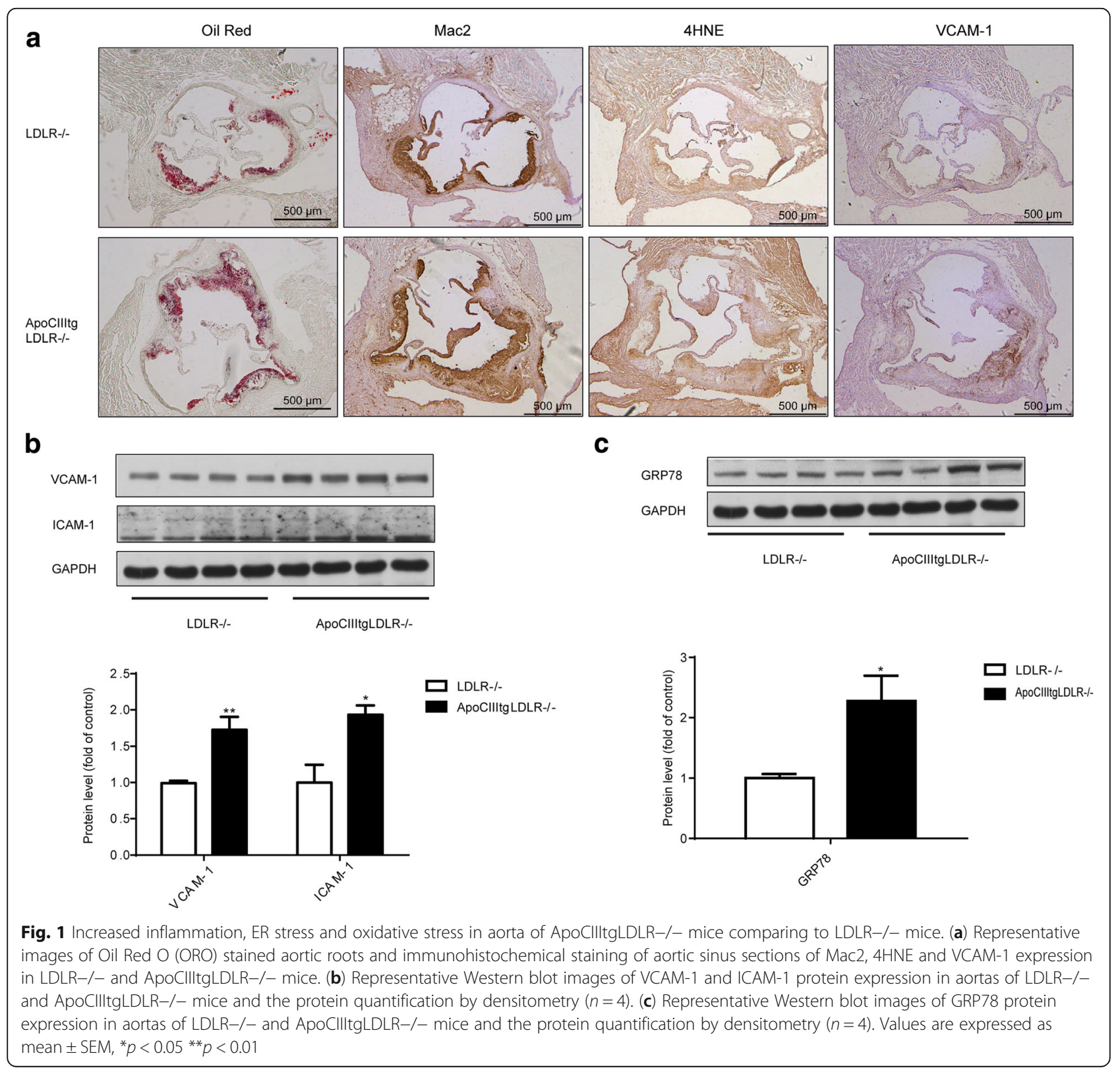


$[6,7]$. Therefore, we examined whether authentic TRLs with or without ApoCIII have pro-inflammatory effects. Because the triglyceride level in the plasma of ApoCIII-/mice is low, it is difficult to obtain the TRLs without ApoCIII (TRLs-ApoCIII). Therefore, we crossed ApoCIIItg mice and ApoCIII-/- mice with GPIHBP1-/- mice, another extreme hypertriglyceridemia mouse model, to generate two hyperlipidemia mice models: ApoCIIItgGPIHBP1-/- and ApoCIII/GPIHBP1 DKO mice, respectively. TRLs+/-ApoCIII were then separated from these two mice models. We found that TRLs+ApoCIII could induce MCP1 expression in HUVECs (Fig. 2a) and under the stimulation of $1 \mu \mathrm{g} / \mathrm{ml}$ LPS, TRLs+ApoCIII can also promote the expression of VCAM-1, reflecting the proinflammatory effects (Fig. 2b). Consistently, comparing to TRLs-ApoCIII, TRLs+ApoCIII promots the expression of IL6 and MCP1 also in peritoneal macrophages (Fig. 2c). These data suggested TRLs could aggravate inflammation depending on ApoCIII.

\section{ER stress and oxidative stress in HUVECs cells}

In vivo data showed increased expression of ER stress related proteins in ApoCIIItg mouse aorta (Fig. 1c). To investigate the effect of TRLs on ER stress in HUVECs cells, we used different doses of TRLs to incubate HUVECs. The result showed that TRLs-ApoCIII induces the expression of oxidative stress and ER stress related proteins in a dose-dependent fashion (Fig. 3a and b), although there was no significant difference between two types of TRLs (Fig. 3c and d). Therefore, our results established that TRLs increase oxidative stress and ER stress level independent of ApoCIII in HUVECs cells.

\section{ER stress and oxidative stress in macrophages}

To investigate the effect of TRLs on oxidative stress and ER stress in macrophages, we incubated rat VSMCs and mouse peritoneal macrophage cells with TRLs. TRLs +/-ApoCIII markedly increased the protein expression of PDI (protein disulfide isomerase) and PERK in VSMCs, but there was no significant difference between the two types of TRLs (Fig. 4a). Similarly, both types of TRLs promote the expression of the proteins related to oxidative stress in macrophage cells, and there was no significant difference between these two TRLs types (Fig. 4b). However, unlike endothelial cells and VSMCs, no TRLs could induce the expression of GRP78,
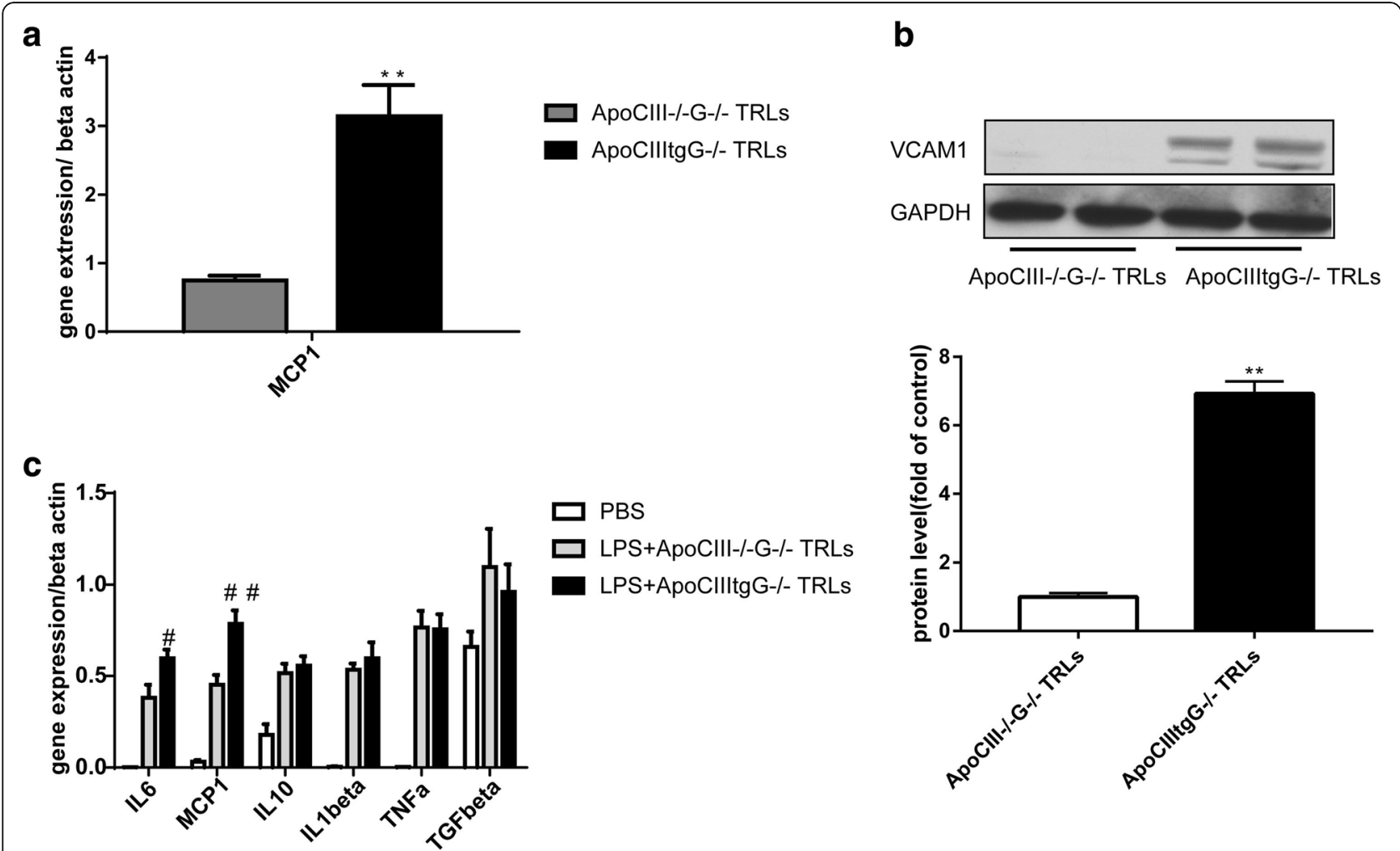

Fig. 2 TRLs increase inflammation in HUVECs and macrophages dependent on ApoCIII. (a) MCP1 expression in HUVECs incubated with 100 $\mu$ g/ml TRLs for $48 \mathrm{~h}(n=4),{ }^{* *} p<0.01$. (b) Western blot images (up) and the protein quantification (down) of VCAM-1 in HUVECs after the incubation with $20 \mu \mathrm{g} / \mathrm{ml}$ TRLs for $24 \mathrm{~h}$ and $1 \mu \mathrm{g} / \mathrm{ml}$ LPS for $6 \mathrm{~h}$ before the analysis $(n=4)$. (c) Expression of inflammation related genes in peritoneal macrophages after the incubation with $100 \mu \mathrm{g} / \mathrm{ml}$ TRLs for $24 \mathrm{~h}$ and an $1 \mu \mathrm{g} / \mathrm{ml}$ LPS stimulation for the last $6 \mathrm{~h}(n=4)$ 


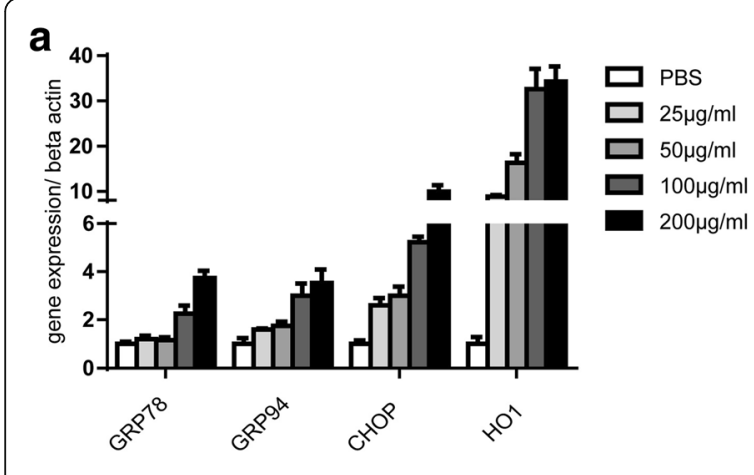

C

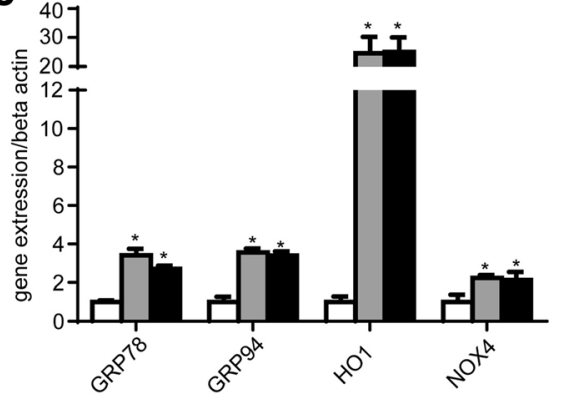

b

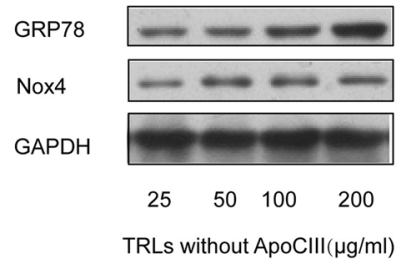

$\square$ PBS
ApoCIII-/-G-/-TRLs
ApoCIIItgG-/-TRLs

d

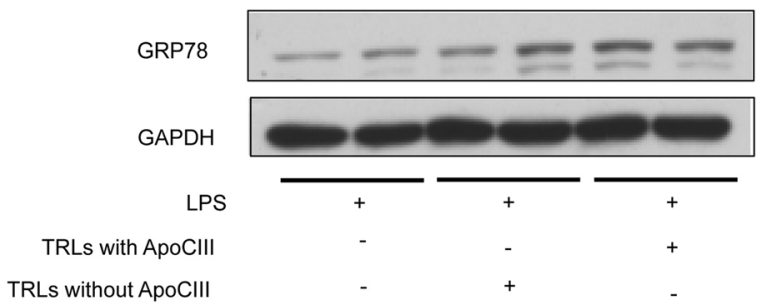

Fig. 3 TRLS increase ER stress and oxidative stress in HUVECs independent of ApoCIII. (a) Expression of ER stress related genes in HUVECs after the incubation of different doses of TRLs without ApoCIII $(n=4)$. (b) Proteins related to ER stress in HUVECs after the incubation of different doses of TRLs without ApoCIII $(n=4)$. (c) Expression of ER stress related genes in HUVECs after the incubation of TRLs without or without ApoCIII $(n=4)$. ${ }^{*} p<0.05$ vs. PBS. (d) Western blot images of GRP78 after the incubation of TRLs with or without ApoCIII $(n=4)$

GRP94, and HO-1 genes, suggesting that TRLs have no effect on ER stress in macrophages (Fig. 4c and d).

\section{Discussion}

Zilversmit first proposed that postprandial hyperlipidemia may play an important role in atherosclerosis thirty years ago [16]. In recent years, lines of evidence from epidemiological investigations, clinical and experimental research emerged showing that non-fasting TRLs level as a risk factor for atherosclerosis and cardiovascular disease [17]. "Residual risk" in statin therapy also supports TRLs as a risk factor in atherosclerosis [18].

TRLs can indirectly promote atherosclerosis. Hypertriglyceridemia is often associated with the atherosclerosis prone lipid profile. Increased small dense LDL is much more easily oxidized and adhered to the vessel wall, and is harder to remove; and increased lipid transferring to TRLs from HDL leads to lower HDL cholesterol $[19,20]$. Elevated triglycerides, small dense LDL and reduced HDL form the lipid distribution of atherosclerosis.

TRLs can also directly promote atherosclerosis. It has shown that remnant like lipoprotein (RLP) in human atherosclerotic plaques and chylomicron remnants (CMR) enter and remain in the blood vessel wall. CMR and TRLs contribute to endothelial dysfunction, macrophage foam cell formation, and the proliferation of vascular smooth muscle cells [21]. TRLs can increase the expression of coagulation factor VII and plasminogen activator inhibitor I (PAI-1) therefore promoting coagulation, including platelet aggregation [22].

As the dominating apolipoprotein in TRLs, whether ApoCIII directly promotes atherosclerosis still remains to be determined. Here, to separate the effect of ApoCIII and TRLs, we isolated biologically active TRLs+/-ApoCIII from the plasma of ApoCIIItgGPIHPB1-/- and ApoCIII/GPIHBP1 DKO mice. Our previous data showed that TRLs could induce proliferation and inflammation in VSMCs dependent on ApoCIII [12]. In the present study, we mainly focused on the endothelial cells and macrophages.

In vivo immunohistochemistry and Western blot experiments showed that ApoCIII overexpression increases the inflammation level in aorta. In our in vitro experiments, we used the natural TRLs+/-ApoCIII and confirmed that ApoCIII can promote the expression of inflammatory factors in endothelial cells and macrophages. The pro-inflammation effect of ApoCIII involves the Akt pathway in VSMCs as we reported previously [12], and the mechanism of ApoCIII in endothelial cell needs to be investigated in the future.

Compared to control mice, ApoCIIItg mice showed higher ER stress level in aorta (Fig. 1c). Incubated with TRLs, HUVECs showed dose dependent increase of ER stress level (Fig. 3a and b). However, there was no 


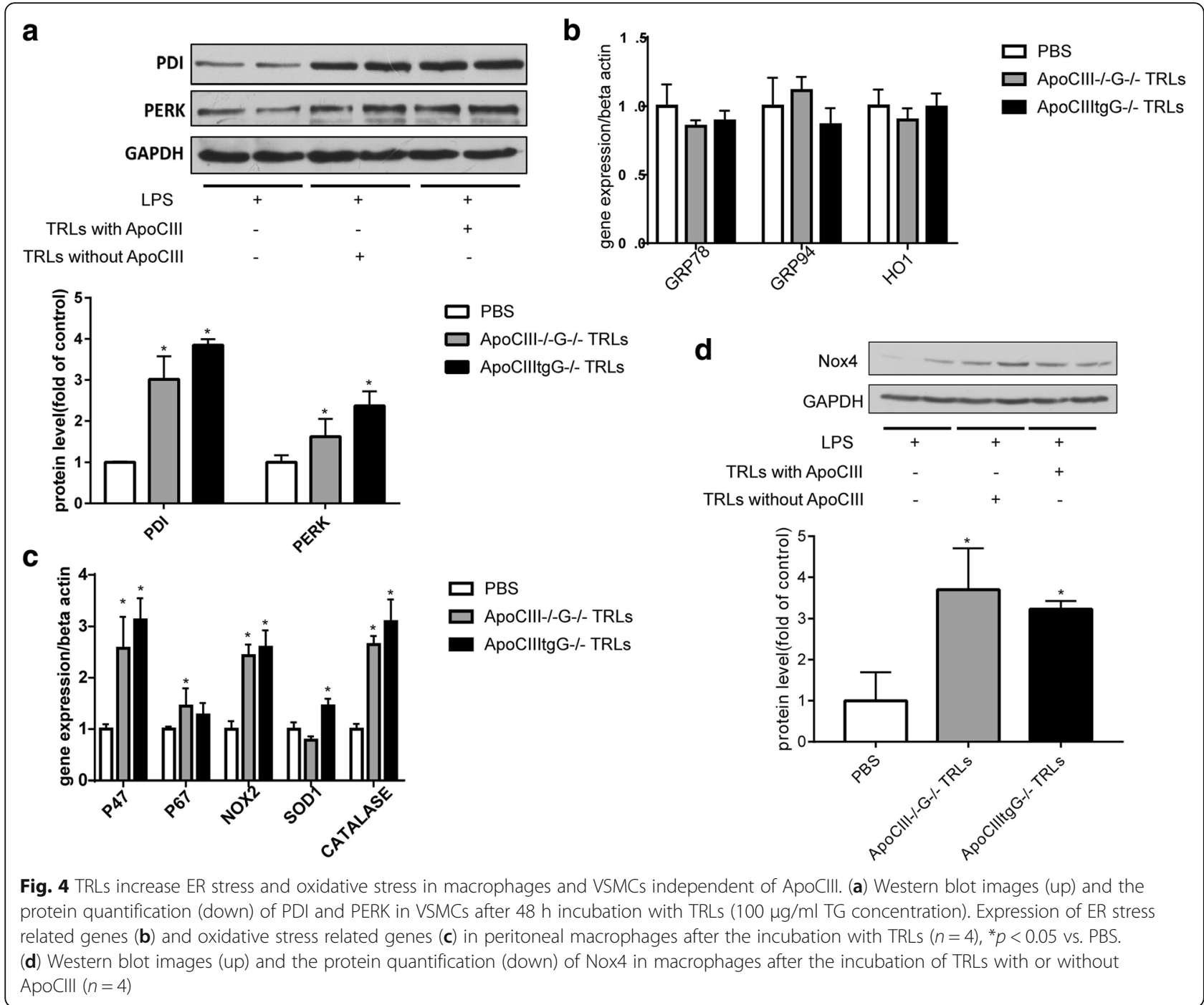

difference between effects of TRLs with and without ApoCIII (Fig. 3c and d). It has been reported that TRLs induce ER stress and oxidative stress in endothelial cells [23]. Here, we confirmed that, unlike inflammation, ER stress and oxidative stress are induced by TRLs independent of ApoCIII.

A targeted approach to reduce plasma levels of ApoCIII can be achieved by providing an antisense inhibitor of ApoCIII synthase (ApoCIII-ASO) [24-26]. When people with severe hypertriglyceridemia were treated with ApoCIII-ASO, the resulting decrease of ApoCIII in the plasma was accompanied by a major reduction of triglyceride in the plasma and a substantial increase of HDL-C [25]. However, the effects of inhibiting ApoCIII synthesis on ASCVD risk are not known.

\section{Conclusions}

In conclusion, our data showed that ApoCIII promotes inflammation in endothelial cells and macrophages and that TRLs from ApoCIII induced HTG could lead to high ER stress level in endothelial cells which may contribute to the progression of atherosclerosis. These results suggest that ApoCIII-ASO may represent a novel therapeutic approach against ASCVD.

\section{Abbreviations}

4HNE: 4-Hydroxynonenal; ApoAV: Apolipoprotein AV; ApoCIl: Apolipoprotein Cll; ApoCIII: Apolipoprotein CIII; ApoCIII-ASO: ApoClll-antisense oligonucleotide; ASCVD: Arteriosclerotic cardiovascular disease; CHOP: CCAAT/enhancerbinding protein homologous protein; CMR: Chylomicron remnants; CMs: Chylomicrons; DAB: Diaminobenzidine; DKO: Double knockout; ER: Endoplasmic reticulum; FBS: Fetal bovine serum; GAPDH: Glyceraldehyde 3-phosphate dehydrogenase; GPIHBP1: Glycosylphosphatidylinositol high density lipoprotein-binding protein 1; GRP78: Glucose regulated protein 78; GRP94: Gloucose requlated protein 94; HO-1: Heme oxygenase-1;

HTG: Hypertriglyceridemia; HUVECs: Human umbilical vein endothelial cells; ICAM-1: Intercellular Adhesion Molecule 1; IL1: Interleukin 1; IL10: Interleukin 10; IL6: Interleukin 6; LDL: Low density lipoprotein; LDLR: Low density lipoprotein receptor; LPL: Lipoprotein lipase; LPS: Lipopolysaccharide; MCP1: Monocyte chemotactic protein 1; Nox4: NADPH oxidase 4; OCT: Optimal cutting temperature; ORO: Oil red O; P47: NADPH oxidase p47; P67: NADPH oxidase p67; PBS: Phosphate-buffered saline; PDI: Protein 
disulfide isomerase; PERK: Protein kinase R (PKR)-like endoplasmic reticulum kinase; PKC: Protein kinase C; RLP: Remnant like lipoprotein; SOD1: Superoxide dismutase1; TGFbeta: Transforming growth factor- $\beta$; TNF: Tumor Necrosis Factor; TRLs: Triglyceride-rich lipoproteins; VCAM-1: Vascular cell adhesion molecule 1; VLDL: Very lowdensity lipoproteins; VSMCs: Vascular smooth muscle cells

\section{Acknowledgements}

We would like to thank Yuhui Wang (Institute of Cardiovascular Science, Peking University) for expertise and equipment; and George Liu (Institute of Cardiovascular Science, Peking University) for advice and suggestions on the manuscript.

\section{Funding}

This study was supported by the National Natural Science Foundation for Young Scientists awarded to Xiaohong Zhang (No. 81300228) and the Excellent Scholars of Beijing Dongcheng District awarded to Yahong $\mathrm{Ma}$ (No. 2014074181449G008)

\section{Availability of data and materials}

All data generated or analyzed during this study are included within the article.

\section{Authors' contribution}

$\mathrm{YH}$ carried out the animals experiments and drafted the manuscript. YM performed the cell culture and Western blot. JW finished the molecular biology experiment and $\mathrm{XH}$ isolated the TRLs. XZ planned the study protocol, coordinated the research, revised and prepared the final version of the manuscript. All authors read and approved the final manuscript.

\section{Ethics approval}

The animal protocol was approved by the Ethics Committee of Peking University Health Science Center (Beijing, China).

\section{Consent for publication}

All authors agree to publish this article in the journal of Lipids in Health and Disease.

\section{Competing interests}

The authors declare that they have no competing interests.

\section{Publisher's Note}

Springer Nature remains neutral with regard to jurisdictional claims in published maps and institutional affiliations.

\section{Author details}

${ }^{1}$ Beijing Anzhen Hospital of Capital Medical University and Beijing Institute of Heart Lung and Blood Vessel Diseases, Beijing 100029, China. ${ }^{2}$ Department of Endocrinology, Beijing Puren Hospital, Beijing 100062, China. ${ }^{3}$ Department of Laboratory Medicine, Beijing Tongren Hospital, Capital Medical University, Beijing 100730, China.

Received: 10 July 2018 Accepted: 11 September 2018

\section{Published online: 17 September 2018}

\section{References}

1. Usman A, Ribatti D, Sadat U, Gillard JH. From lipid retention to immunemediate inflammation and associated angiogenesis in the pathogenesis of atherosclerosis. J Atheroscler Thromb. 2015;22:739-49.

2. Witztum JL, Steinberg D. Role of oxidized low density lipoprotein in atherogenesis. J Clin Invest. 1991;88:1785-92.

3. Nordestgaard BG, Benn M, Schnohr P, Tybjaerg-Hansen A. Nonfasting triglycerides and risk of myocardial infarction, ischemic heart disease, and death in men and women. JAMA. 2007;298:299-308.

4. Bansal S, Buring JE, Rifai N, Mora S, Sacks FM, Ridker PM. Fasting compared with nonfasting triglycerides and risk of cardiovascular events in women. JAMA. 2007:298:309-16.

5. Sarwar N, Danesh J, Eiriksdottir G, Sigurdsson G, Wareham N, Bingham S, Boekholdt SM, Khaw KT, Gudnason V. Triglycerides and the risk of coronary heart disease: 10,158 incident cases among 262,525 participants in 29 Western prospective studies. Circulation 2007;115:450-8

6. Kawakami A, Aikawa M, Libby P, Alcaide P, Luscinskas FW, Sacks FM. Apolipoprotein CIII in apolipoprotein B lipoproteins enhances the adhesion of human monocytic cells to endothelial cells. Circulation. 2006;113:691-700.

7. Kawakami A, Aikawa M, Alcaide P, Luscinskas FW, Libby P, Sacks FM. Apolipoprotein CIII induces expression of vascular cell adhesion molecule-1 in vascular endothelial cells and increases adhesion of monocytic cells. Circulation. 2006;114:681-7.

8. Voshol PJ, Rensen PC, van Dijk KW, Romijn JA, Havekes LM. Effect of plasma triglyceride metabolism on lipid storage in adipose tissue: studies using genetically engineered mouse models. Biochim Biophys Acta. 2009;1791:479-85.

9. Ginsberg HN, Le NA, Goldberg IJ, Gibson JC, Rubinstein A, Wang-Iverson P, Norum R, Brown WV. Apolipoprotein B metabolism in subjects with deficiency of apolipoproteins CIII and Al. Evidence that apolipoprotein CIII inhibits catabolism of triglyceride-rich lipoproteins by lipoprotein lipase in vivo. J Clin Invest. 1986:78:1287-95.

10. Windler $E$, Havel RJ. Inhibitory effects of $C$ apolipoproteins from rats and humans on the uptake of triglyceride-rich lipoproteins and their remnants by the perfused rat liver. J Lipid Res. 1985;26:556-65.

11. Qin W, Sundaram M, Wang Y, Zhou H, Zhong S, Chang CC, Manhas S, Yao EF, Parks R, McFie PJ, Stone SJ, Jiang ZG, Wang C, Figeys D, Jia W, Yao Z. Missense mutation in APOC3 within the C-terminal lipid binding domain of human ApoC-III results in impaired assembly and secretion of triacylglycerol-rich very low density lipoproteins: evidence that ApoC-III plays a major role in the formation of lipid precursors within the microsomal lumen. J Biol Chem. 2011;286:27769-80.

12. Li H, Han Y, Qi R, Wang Y, X Zhang MY, Tang Y, Wang M, Shu YN, Huang W, Liu X, Rodrigues B, Han M, Liu G. Aggravated restenosis and atherogenesis in ApoCIII transgenic mice but lack of protection in ApoCIII knockouts: the effect of authentic triglyceride-rich lipoproteins with and without ApoCIII. Cardiovasc Res. 2015:107:579-89.

13. Jorgensen AB, Frikke-Schmidt R, Nordestgaard BG, Tybjaerg-Hansen A. Lossof-function mutations in $\mathrm{APOC} 3$ and risk of ischemic vascular disease. N Engl J Med. 2014;371:32-41.

14. Tg NHL. Hdl working Group of the Exome Sequencing Project, I. blood, J Crosby, GM Peloso, PL Auer, DR Crosslin, NO Stitziel: loss-of-function mutations in APOC3, triglycerides, and coronary disease. N Engl J Med. 2014;371:22-31.

15. Ross R. Atherosclerosis is an inflammatory disease. Am Heart J. 1999;138: S419-20.

16. Zilversmit DB. Atherogenesis: a postprandial phenomenon. Circulation. 1979; 60:473-85.

17. Shapiro MD, Fazio S. From lipids to inflammation: new approaches to reducing atherosclerotic risk. Circ Res. 2016;118:732-49.

18. HN Hodis WJ, Mack SP, Azen P, Alaupovic JM, Pogoda L, LaBree LC, Hemphill DM, Kramsch DH. Blankenhorn: triglyceride- and cholesterol-rich lipoproteins have a differential effect on mild/moderate and severe lesion progression as assessed by quantitative coronary angiography in a controlled trial of lovastatin. Circulation. 1994;90:42-9.

19. Stalenhoef AF, de Graaf J. Association of fasting and nonfasting serum triglycerides with cardiovascular disease and the role of remnant-like lipoproteins and small dense LDL. Curr Opin Lipidol. 2008;19:355-61.

20. Han Y, Qi R, Liu G. Reduced high density lipoprotein cholesterol in severe hypertriglyceridemic ApoCIII transgenic mice via lowered hepatic ApoAl synthesis. Biochem Biophys Res Commun. 2015;462:420-5.

21. Botham KM, Wheeler-Jones CP. Postprandial lipoproteins and the molecular regulation of vascular homeostasis. Prog Lipid Res. 2013;52:446-64.

22. Eriksson P, Nilsson L, Karpe F, Hamsten A. Very-low-density lipoprotein response element in the promoter region of the human plasminogen activator inhibitor-1 gene implicated in the impaired fibrinolysis of hypertriglyceridemia. Arterioscler Thromb Vasc Biol. 1998;18:20-6.

23. Wang YI, Bettaieb A, Sun C, DeVerse JS, Radecke CE, Mathew S, Edwards CM, Haj FG, Passerini AG, Simon SI. Triglyceride-rich lipoprotein modulates endothelial vascular cell adhesion molecule (VCAM)-1 expression via differential regulation of endoplasmic reticulum stress. PLoS One. 2013;8:e78322.

24. Graham MJ, Lee RG, Bell TA, Fu W, Mullick AE, Alexander VJ, Singleton W, Viney N, Geary R, Su J, Baker BF, Burkey J, Crooke ST, Crooke RM. Antisense 
oligonucleotide inhibition of apolipoprotein C-III reduces plasma triglycerides in rodents, nonhuman Primates, and humans. Circ Res. 2013;112:1479-90.

25. Gaudet D, Brisson D, Tremblay K, Alexander VJ, Singleton W, Hughes SG, Geary RS, Baker BF, Graham MJ, Crooke RM, Witztum JL. Targeting APOC3 in the familial chylomicronemia syndrome. N Engl J Med. 2014;371:2200-6.

26. Gordts PL, Nock R, Son NH, Ramms B, Lew I, Gonzales JC, Thacker BE, Basu D, Lee RG, Mullick AE, Graham MJ, Goldberg IJ, Crooke RM, Witztum JL, Esko JD. ApoC-III inhibits clearance of triglyceride-rich lipoproteins through LDL family receptors. J Clin Invest. 2016;126:2855-66.

Ready to submit your research? Choose BMC and benefit from:

- fast, convenient online submission

- thorough peer review by experienced researchers in your field

- rapid publication on acceptance

- support for research data, including large and complex data types

- gold Open Access which fosters wider collaboration and increased citations

- maximum visibility for your research: over $100 \mathrm{M}$ website views per year

At $\mathrm{BMC}$, research is always in progress.

Learn more biomedcentral.com/submissions 